\title{
An abdominal cyst
}

\author{
GL Smith, PA Thorpe, $S$ Karimjee, PM Dawson
}

\section{West Middlesex} University Hospital NHS Trust, London, UK

Department of

Surgery,

GL Smith

PM Dawson

Department of

Histopathology

PA Thorpe

Department of

Radiology

$S$ Karimjee

Correspondence to

Mr PM Dawson, Consultant

Surgeon, West Middlesex

University Hospital NHS

Trust, Twickenham Road

Isleworth, Middlesex

TW7 6AF, UK

Accepted 19 April 1995

\section{Case Report}

A 29-year-old woman presented with a 12-month history of abdominal swelling. On examination, there was a smooth cystic mass arising from the pelvis and occupying the lower abdomen. Vaginal examination was normal. A computed tomography (CT) scan was performed (figure 1).

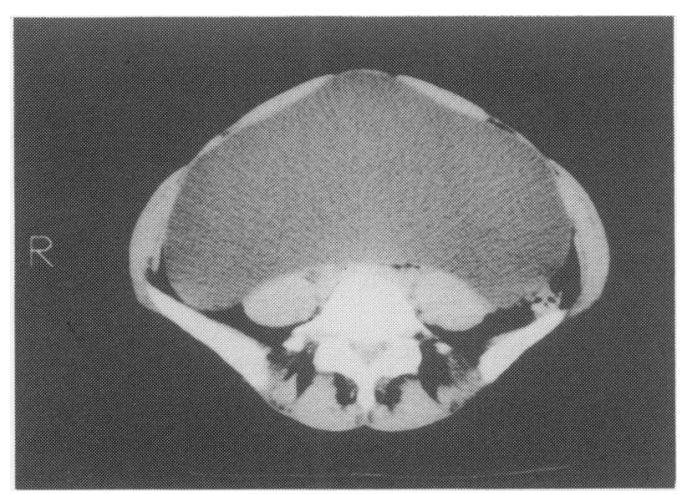

\section{Question}

Suggest two differential diagnoses
Figure 1 CT scan showing a large unilocular cyst. The right ovary was normal; the left was not seen 


\section{Answer}

\section{Ovarian or mesenteric cyst}

The patient underwent a laparotomy at which a 5-litre cyst was excised from the sigmoid mesocolon (figure 2). The ovaries were normal. Histology revealed a urogenital mesenteric cyst of the mullerian type (figure 3). The patient made an uneventful recovery.

\section{Comment}

Mesenteric urogenital cysts are well described but rare abnormalities thought to be derived from vestigial remnants of the embryonic urogenital apparatus. ${ }^{1}$ They are distinct from lymphatic and enteric cysts found in similar locations. Despite their developmental origin, they usually present in adults rather than children. ${ }^{2}$

Pre-operative diagnosis of such cysts is often difficult. Ultrasound and CT scanning may be suggestive, but it is often impossible to make an accurate distinction from other (more common) pathological entities, particularly ovarian cysts. In this case, the normal findings on vaginal examination raised suspicion that the cyst was not arising from the gynaecological organs.

Mesenteric urogenital cysts have been classified into pronephric, mesonephric, metanephric and-mullerian variants, on the basis of their histology and anatomical location, although it is unusual for them to fall definitively into any of these categories. The mullerian type have characteristic ciliated columnar epithelium resembling that of normal fallopian tube and are particularly rare. Only two other cases have been described in the literature. . $^{3,4}$

In most cases, as in this patient, the blood supply is not derived directly from major abdominal vessels. ${ }^{3}$ Complete excision is usually possible without jeopardising the vascularity of adjacent organs. Malignant change or recurrence after complete excision have never been described and surgical removal is therefore curative.

\section{Final diagnosis}

Mullerian urogenital mesenteric cyst arising in the sigmoid mesocolon

1 Handfield-Jones RM. Retroperitoneal cysts: their pathology, diagnosis and treatment. Br $\mathcal{F}$ Surg 1924; 12: 119-34

2 Shandalakis JE. Mesenteric cyst: a reports of three cases. $f$ Med Assoc Ga 1955; 44: 75-80.

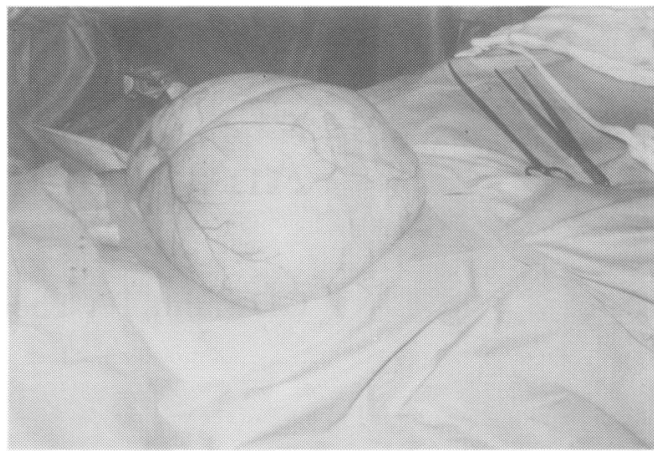

Figure 2 The cyst revealed at laparotomy

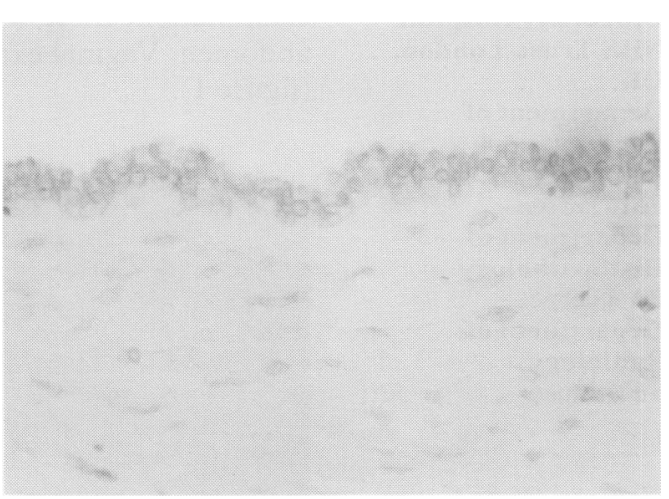

Figure 3 The cyst was lined with ciliated columnar epithelium resembling that of fallopian tube. The wall was fibrous

\section{Learning points}

- mesenteric urogenital cysts are rare developmental abnormalities

- they present in adults rather than children

- the mullerian type has characteristic columnar ciliated epithelium resembling that of normal fallopian tube and is exceptionally rare

- ultrasound and CT scanning may not accurately distinguish mesenteric cysts within the pelvis from ovarian cysts

- confirmation of the diagnosis and curative treatment are both achieved by surgical exploration and excision

Keywords: mesenteric urogenital cyst, computed tomography

3 Harpaz N, Gellman E. Urogenital mesenteric cyst with fallopian tubal features. Arch Pathol Lab Med 1987; 111: 78-80.

4 Steinberg L, Rothman D, Drey NW. Mullerian cyst of the

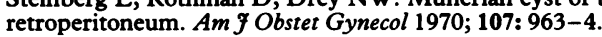

